\title{
Dificuldades encontradas pela família no cuidado à criança/adolescente com HIV
}

\author{
Difficulties encountered by families in caring for children or adolescents with HIV \\ Dificultades encontradas por la familia en el cuidado al niño/adolescente con VIH
}

\author{
Aline Rodrigues Costal' Camila Magroski Goulart Nobre"'; Giovana Calcagno Gomes"II; \\ Simone Quadros Alvarez ${ }^{\text {; }}$; Juliane Portella Ribeiro ${ }^{v}$; Gabriela Silva Miranda Rosa ${ }^{v}$
}

\begin{abstract}
RESUMO
Objetivo: conhecer as dificuldades encontradas pela família para o cuidado à criança/adolescente com HIV. Metodologia: pesquisa qualitativa realizada em um Hospital Dia no sul do Brasil em 2018. Participaram 20 familiares cuidadores de crianças/adolescentes soropositivas. Os dados foram coletados por entrevistas e submetidos à Análise de Conteúdo. Respeitaram-se os princípios éticos. Resultados: as famílias afirmaram não ter facilidades no cuidado. Referiram dificuldades em manter o sigilo do diagnóstico para a criança/adolescente; na adesão desta à medicação e minimização dos seus efeitos colaterais, em seguir os horários de administração; na assiduidade às consultas; em sofrer preconceito e na aceitação da sociedade diante da doença, incluindo a preocupação com o futuro da criança/adolescente. Conclusão: verificou-se como importante a atuação dos profissionais da saúde/ enfermeiros investindo em uma assistência voltada para a promoção de saúde de crianças e adolescentes com HIV, promovendo educação em saúde e o manejo das dificuldades enfrentadas com o cuidado. Descritores: Criança; adolescente; família; HIV; enfermagem.
\end{abstract}

\section{ABSTRACT}

Objective: to learn the difficulties encountered by families in caring for children or adolescents with HIV. Methodology: this qualitative study was conducted at a day hospital in southern Brazil in 2018. The participants were 20 family caregivers of seropositive children or adolescents. Data were collected through interviews and subjected to Content Analysis. Ethical principles were respected. Results: the families claimed to have no care facilities. They reported difficulties in keeping the children's or adolescents' diagnosis confidential; securing adherence to medication and administration schedules; minimizing side effects; attending appointments; suffering prejudice and gaining social acceptance of the disease, which included concern for the children's or adolescents' futures. Conclusion: it was found to be important that health professionals' or nurses' invest in care focused on promoting the health of children and adolescents with HIV, furthering health education and managing the difficulties encountered in care.

Descriptors: Child; adolescent; family; HIV; nursing.

\section{RESUMEN}

Objetivo: conocer las dificultades encontradas por la familia para el cuidado al niño/adolescente con VIH. Método: investigación cualitativa realizada en un Hospital Día en el sur de Brasil en 2018. Participaron 20 familiares cuidadores de niños/adolescentes seropositivos. Los datos fueron recolectados por entrevistas y sometidos al Análisis de Contenido. Se respetaron los principios éticos. Resultados: las familias afirmaron no tener facilidades en el cuidado. Dijeron que tienen dificultades para mantener e secreto del diagnóstico junto al niño/adolescente, también para su adherencia a la medicación y minimización de sus efectos colaterales, para seguir los horarios correctos de administración; la asiduidad de las consultas; por sufrir con los prejuicios y la dificultad de aceptación social de la enfermedad y la preocupación por el futuro del niño/adolescente. Conclusión: se verificó que es muy importante la actuación de los profesionales de salud/enfermeros que invierten en una atención volcada a la promoción de salud de niños y adolescentes con VIH, promoviendo la educación sanitaria y la gestión de las dificultades enfrentadas con el cuidado.

Descriptores: Niño; adolescente; familia; HIV; enfermería.

\section{INTRODUÇÃO}

Os cuidados prestados à criança e o adolescente diagnosticado com HIV são variados e necessitam da atenção e da disponibilidade do familiar cuidador. É essa família que acompanha a criança/adolescente durante todo seu crescimento e desenvolvimento, tendo experiências e vivências que lhes possibilitam construir cuidados que a auxiliam viver com qualidade. Portanto, é importante que a enfermagem se comprometa em fornecer uma assistência e permanecer junto dessas famílias, atuando e auxiliando-as na construção do cuidado à criança/adolescente para que ela perpasse as dimensões humanas conforme o seu contexto e o modo de vida ${ }^{1}$.

'Doutoranda de Enfermagem na Universidade Federal do Rio Grande. Rio Grande: Rio Grande do Sul: Brasil. E-mail: aline.rodrigues.costa@hotmail.com.

"Doutoranda de Enfermagem na Universidade Federal do Rio Grande. Rio Grande: Rio Grande do Sul: Brasil. E-mail: kamy_magroski@yahoo.com.br.

I'Doutora em Enfermagem. Docente da Universidade Federal do Rio Grande. Brasil. E-mail: giovanacalcagno@furg.br.

IV Doutora em Enfermagem na Universidade Federal do Rio Grande. Rio Grande. Rio Grande do Sul: Brasil. E-mail: alvarez.sqa@gmail.com.

voutora em Enfermagem na Universidade Federal do Rio Grande. Professora Adjunta, Universidade Federal de Pelotas. Brasil. E-mail: ju_ribeiro1985@hotmail.com.

v'Acadêmica de Enfermagem na Universidade Federal do Rio Grande. Rio Grande: Rio Grande do Sul: Brasil. E-mail: gabrielasilva.enf@outlook.com. 
Ao longo do tempo a família observa, descobre, aprende a desenvolver e fornecer os cuidados. Precisa e adquire atenção frente as necessidades e as coisas que acontecem para se adaptar aos cuidados da criança/adolescente. Fica tranquila quando a mesma aceita o remédio, aprende que tem que tomar a medicação, lembra dos horários, solicita o medicamento, começa a tomar sozinha e participar do seu próprio cuidado².

A família é quem normalmente cuida e fornece grande parte do suporte necessário para a criança/adolescente durante o seu desenvolvimento. Entretanto, nesta caminhada podem surgir dificuldades que interferem nas suas vidas. Observaram-se dificuldades que geram problemas no acompanhamento periódico da criança/adolescente ao Serviço de Assistência Especializada (SAE) ${ }^{3}$. A principal foi quanto ao acesso entre a residência e o SAE na qual o familiar cuidador dependia de recursos e apoio instrumental para custear o transporte. A mesma dificuldade foi também citada fora do Brasil. Em Uganda, África também foi citada a falta de transporte para ir aos serviços de saúde como uma barreira dificultadora ${ }^{4}$. Além disso, também alegam dificuldade na marcação de consultas, devido aos tipos de encaminhamentos necessários para o agendamento, o que dispendia um período de tempo maior ${ }^{3}$.

A ausência de uma renda financeira estável e as condições de vulnerabilidades que muitas famílias se encontram, afetam a forma como o seu cuidado é realizado. Constatou-se em Uganda, que a falta de um suporte financeiro para custear os alimentos adequados e nutritivos para a criança/adolescente foi mencionado como uma dificuldade que interfere na sua qualidade. Além disso, declararam possuir dificuldades no cuidado quando a mesmo se encontrava doente ${ }^{4}$.

Diante das diversas dificuldades que as famílias enfrentam, a figura de um profissional que se faça presente e que auxilie durante todo esse processo de adaptação da criança/adolescente é essencial. 0 enfermeiro é o primeiro profissional que entra em contato com os pacientes com HIV e suas famílias, portanto, é importante que o mesmo utilize os conhecimentos aprendidos ao longo da sua prática para criar estratégias que minimizem as dificuldades e fragilidades que possam ser enfrentadas. Ademais, a criação do vínculo com a criança/adolescente é essencial para que a confiança, a intimidade, a empatia e a afetividade sejam estabelecidas e o enfermeiro consiga orienta-la 5 .

Deste modo, o objetivo do estudo é conhecer as dificuldades encontradas pela família para o cuidado à criança/adolescente com HIV. Acredita-se que o estudo ampliará o conhecimento dos profissionais da saúde frente a temática, de forma a auxiliar na criação de estratégias que facilitem as famílias no cuidado à criança/adolescente soropositivo.

\section{METOdologiA}

Foi conduzido por uma abordagem qualitativa do tipo descritiva e exploratória. Teve como contexto o Hospital Dia Pediátrico de um Hospital Universitário localizado no sul do Brasil. Os participantes deste estudo foram 20 familiares cuidadores de crianças e adolescentes acompanhados no Hospital Dia, no período da coleta de dados. Atenderam ao critério de inclusão: ser o principal familiar cuidador da criança/adolescente com HIV no domicílio, acompanhar a criança/adolescente periodicamente no tratamento no Hospital Dia e ter 18 anos ou mais. Como critério de exclusão foi utilizado acompanhar a criança/adolescente eventualmente nas consultas.

A coleta de dados foi realizada no primeiro semestre de 2018 por meio de uma entrevista individual com cada familiar cuidador realizada em um consultório individual do Hospital Dia após a consulta médica ou no momento de espera da mesma. O número de participantes do estudo foi definido pela saturação de dados. Foram questionados acerca das dificuldades enfrentadas para cuidar a criança/adolescente com HIV. Os dados coletados foram analisados pela técnica de Análise de conteúdo. A análise de conteúdo é uma análise dos "significados", pois este método enriquece a tentativa exploratória, e aumenta a propensão para a descoberta sendo um método empírico ${ }^{6}$. Esta técnica foi operacionalizada através de três etapas: na pré análise realizou-se a leitura flutuante dos dados e a escolha dos depoimentos mais significativos que ilustrassem o conteúdo das categorias e a preparação do material; na exploração do material foram definidas as categorias e no tratamento dos resultados obtidos e interpretação os dados foram discutidos a partir de autores estudiosos da temática ${ }^{6}$.

Foi respeitada a Resolução 466/12 que rege as pesquisas envolvendo seres humanos. O projeto de pesquisa foi submetido ao Comitê de Ética em Pesquisa na Área da Saúde - e aprovado sob número 29/2018. Os participantes assinaram o Termo de Consentimento Livre e Esclarecido e foram identificados pela letra $F$ de familiar, seguida do número da entrevista para garantir seu anonimato.

\section{RESULtAdDOS}

\section{Caracterização dos Participantes}

Participaram 20 familiares cuidadores com idades entre 21 e 65 anos, com uma média de 37,9 anos. Possuem como grau de parentesco da criança mãe (nove), mãe adotiva (quatro), pai (dois), madrasta (uma), tio (um) e avó (três). 
Tendo como escolaridades, ensino fundamental incompleto (dez), ensino fundamental completo (quatro) e ensino médio completo (quatro), ensino incompleto (um) e ensino superior completo (um). Quanto a situação conjugal, sete são solteiros, oito casados, quatro separados e uma viúva. Vivem com uma renda familiar entre $R \$ 187,00$ e $R \$$ $10.000,00$, com uma média de $\mathrm{R} \$ 1.074,00$, sendo que dois informaram não ter renda. As crianças possuíam idades entre nove meses e 14 anos, com uma média de 7,08 anos, sendo onze do sexo masculino e nove do feminino. Todas adquiriram o vírus HIV por transmissão vertical.

\section{Dificuldade em manter o sigilo acerca do diagnóstico da criança/adolescente para a mesma}

Mesmo sendo uma doença na qual o soropositivo pode ter uma vida como qualquer outra pessoa, realizando o tratamento correto, os familiares cuidadores afirmaram não ver facilidades no cuidado prestado. Referiram dificuldades em manter o sigilo do diagnóstico da criança/adolescente para a mesma, pois querem escolher o momento adequado de lhe revelar.

Não tem nada de fácil (F5).

Não tem facilidade nenhuma. Não é fácil (F11).

Quando entra no consultório, as moças não falam HIV ou que ela tem. Eu pedi, porque ela é muito esperta, sabe tudo. Eu quero escolher a melhor hora para contar o que ela tem (F19).

\section{Dificuldades na adesão da medicação pela criança/adolescente}

Verificou-se que uma das dificuldades encontradas foi a adesão da medicação pela criança/adolescente. Referiram o sabor amargo como motivo para a negação da mesma em aceitar o remédio.

o que eu acho difícil em relação à medicação é o gosto amargo. Ele toma um pouco e bota a metade fora. A doutora disse para mim hoje que eu posso misturar ele com um pouco de gelatina ou com suco de laranja (F5).

No começo é que foi bem difícil porque ela não conseguia engolir e o gosto era ruim. Mas ela já se adaptou (F6).

Para ele tomar os remédios é uma briga por causa do gosto (F7).

Além disso, encontram grande dificuldade em administrar os antirretrovirais para a criança/adolescente, pois são medicações que geralmente causam reações gástricas como dor de estômago, enjoo e vômitos, sendo difícil a sua ingestão. Sua maior preocupação é que a medicação deverá ser utilizada por toda a vida.

A medicação ela teve um pouco de reação, dor de estômago e vômitos. Vim na doutora, expliquei e ela me deu um remedinho para o estômago. Ela só toma a medicação porque ela pensa que é para crescer (F19).

Agora chegando na adolescência é meio complicado para ele. Ele tem os efeitos colaterais dos medicamentos, diarreia, vômitos. É complicado porque tem que tomar para sempre para não adoecer. Ele fica meio retraído, mas eu não o culpo de ser assim por esse problema (F17).

Também se constatou como dificuldade os horários de administração dos medicamentos, o que gera interferência no sono da criança/adolescente. Além disso, dois familiares cuidadores relataram possuir dificuldades em relação a alimentação.

[...] é que às vezes ela está dormindo e eu tenho que acordar para dar a medicação. Ai já quebra o sono, porque são três medicações. Tu dás uma medicação e tu esperas para dar a outra para não causar um problema de estômago [...] interrompe o sono e interfere até com os horários da alimentação (F8).

Só com a comida que ele deu uma parada agora. Não quer comer. Aí eu faço ele comer nem que seja uma colherada, porque ele não pode ficar sem se alimentar (F3).

\section{Dificuldade em ser assíduos às consultas}

A assiduidade às consultas devido ao fato de esquecê-las ou pelo familiar cuidador residir em outra cidade e necessitar viajar até o município foram também referidos como dificuldades. Buscam tratamento em outra cidade como forma de garantir o sigilo acerca da sorologia da criança/adolescente. Já uma das participantes, referiu como dificuldade assumir responsabilidade pelo cuidado, pois antes da criança/adolescente não necessitava ter ou ter que assumi-la sozinha.

As consultas é que às vezes eu me passo. Eu ando esquecida da cabeça. Hoje mesmo só me lembrei quando mexi na carteira e vi que era hoje. Aí, tive que vir correndo. (F3).

O difícil é vir para as consultas, porque eu tenho que viajar da minha cidade até aqui [...](F4).

A doutora, que era a médica dele na nossa cidade me encaminhou para a doutora daqui. É difícil vir todos os meses e, ás vezes, eu me esqueço (F15).

No começo foi difícil, porque eu não estava acostumada. Eu sou usuária de crack, também sou soropositiva, eu sustento eles sozinha. Eu sou garota de programa[...] então, eu me desdobro. Nos primeiros três, quatro meses 
que foram brabos, porque assim, tem tanto de remédio, tem que fazer bombinha, tem que levar nas consultas. Eu não estava acostumada com tanta responsabilidade. Eu sempre fui vida louca [...](F4).

\section{Sofrer preconceito e aceitação da sociedade}

O preconceito e aceitação perante a sociedade foram citados como dificuldades vivenciadas. Dois participantes referiram que a razão de se manterem vivos e fortes é a presença da criança/adolescente, chegando a mencionar o suicídio como alternativa caso tivesse consciência da doença antes do nascimento do filho.

É difícil pelo preconceito. Na escola ninguém sabe. Quando ela falta à aula por algum exame a gente diz que é exame de rotina. Ninguém tem que saber (F9),

Eu sinto o preconceito dos outros onde eu moro. Dizem:_A fulana tem HIV. Os próprios parentes do pai dele são preconceituosos. Daí eu sofro e tenho depressão por causa disso. Tenho que tomar os remédios. Às vezes dá vontade de afundar na droga e desistir (F4).

[...] não é qualquer pessoa que vai te aceitar do jeito que tu és. Eu sei porque depois que eu tive ele eu fui tentar ter um parceiro e fui explicar para ele[...] ai ele pegou e disse que não ia ficar comigo assim, porque não queria se arriscar a pegar[...]. Desde então eu não tive mais ninguém. Têm vezes que eu penso em desistir de tudo e não viver mais porque não é qualquer pessoa que aceita. Mas aí quando eu olho para o meu filho eu me esqueço de tudo. Mas se eu tivesse descoberto antes de ter ele eu já teria me matado na hora porque eu não teria porque viver mesmo [...] Eu olho para ele e ele é que é minha alegria de viver, que me dá forças (F5).

O tempo e a forma como explicar para a criança/adolescente sobre seu diagnóstico foi mencionada como dificuldade. Afirmaram não terem pensado ainda sobre o assunto devido à idade da criança, o medo da sua reação e que sejam culpados pela transmissão da doença. Além disso, são muitas vezes questionados pela criança/adolescente do porquê do uso continuo da medicação.

Ele está crescendo. Está entendendo mais. Então como ele vai aceitar que tem a doença? É difícil. Eu não falei bem para ele sobre o que é. Às vezes ele pergunta se vai ter que tomar o remédio para sempre e eu digo que não sei, vamos ver (F1).

[...] eu imagino todos os dias como eu vou explicar para ele no futuro o que aconteceu. Porque [...] tem uma amiga minha que a mãe dela não fez o tratamento e ela tem também e ela é bem louca, revoltada. Diz que é doente por causa da mãe, que a culpa é dela. Eu vejo isso e fico pensando que meu filho pode ter nojo de mim por eu ter transmitido o virus para ele (F5).

Questões referentes ao desenvolvimento da criança/adolescente e aos futuros namoros foram referidas como geradoras de preocupação, sendo mencionadas como dificuldades encontradas. Preocupam-se com a conduta da mesma para que a doença não seja transmitida para os demais. Além disso, uma das entrevistadas referiu preocupação em orientar a filha quanto ao pré-natal quando for adulta para que não faça com o seu filho o que fizeram com ela.

Eu me preocupo com o crescimento dele, com o namoro, porque hoje em dia é tudo muito rápido (F1).

Eu espero que ele seja honesto com as pessoas, que não faça que nem fizeram comigo e que fale a verdade. Se não quiser falar que ele se cuide para não passar para outra pessoa (F7).

Ela vai ser uma cidadã como qualquer outra, ela vai trabalhar, se Deus permitir vai poder ser mãe. Ela vai fazer um pré-natal para ela não fazer com o filho dela o que fizeram com ela. Então, é isso que eu vou tentar passar para a minha filha, que não é o fim do mundo porque tu tens essa doença (F19).

\section{DISCUSSÃO}

Os familiares que cuidam diariamente de crianças/adolescente com diagnóstico de HIV não observam facilidades em cuidá-las, pois encontram uma série de barreiras que afetam a forma como este cuidado é exercido, principalmente frente à revelação do diagnóstico ${ }^{8}$. Esse momento causa uma série de mudanças que interfere na vida da família, sendo a sua escolha uma decisão difícil. Verificou-se que em muitos casos a omissão da doença ocorre devido à pouca idade e falta de maturidade da criança/adolescente para compreender a sua sorologia. Também optam por esperar a mesma obter responsabilidade pelo seu autocuidado e capacidade emocional para lidar com as questões frente ao HIV ${ }^{7}$.

Os familiares escolhem por manter o sigilo acerca do diagnóstico para criança/adolescente, pois querem escolher o momento adequado para a sua revelação ${ }^{8}$. Assim, para que esse momento não seja árduo, deve envolver os profissionais da saúde, tornando-se uma decisão compartilhada e continuada. Além disso, devem ser observados alguns aspectos como as particularidades do contexto social, faixa etária e maturidade cognitiva ${ }^{9}$.

Constatou-se que outro motivo que leva os familiares cuidadores a postergarem a revelação do diagnóstico à criança/adolescente é que durante a infância a mesma não entende, não pergunta e não recusa o tratamento. Entretanto, com a entrada da criança na adolescência novos questionamentos começam a surgir exigindo respostas e mudanças de atitude do familiar cuidador ${ }^{10}$. Além disso, o estigma da doença, mesmo com toda a informação existente, interfere na revelação do diagnóstico o que pode levar a sua revelação na maioria das vezes durante a fase da 
adolescência, que é considerada um momento especial e delicado na vida de uma pessoa. Fator este muito comum, pois os familiares cuidadores acreditam estar protegendo a criança/adolescente ${ }^{11}$. Segundo estudo analisado, observouse que a partir dos 11 anos as crianças/adolescentes entrevistadas referiram começar a compreender um pouco melhor sobre sua sorologia11.

A revelação do diagnóstico é um marco inicial para a construção da história de vida da criança/adolescente. Contudo, esse momento, embora necessário na sua vida e em seus cuidados gera uma série de reações e sentimentos, muitas vezes negativos, já que se trata de uma doença que interfere em diversos aspectos, como os sociais e emocionais, em virtude do estigma ainda existente na sociedade ${ }^{12}$

A percepção de mundo é diferente e ampliada na adolescência e por isso, a revelação do diagnóstico é necessária e essencial para que ocorra o sucesso do Tratamento Antirretroviral (TARV). O adolescente com HIV precisa conhecer e participar das decisões relacionadas ao processo saúde-doença de forma singular e integral' ${ }^{12}$. Assim, é preciso a presença de um profissional da saúde que o acolha e cuide, assim como do seu familiar cuidador em sua singularidade, indo além da doença para encontrar as particularidades de cada um ${ }^{10}$.

Por ser o HIV na infância uma problemática de difícil compreensão que pode gerar muitas dúvidas e questionamentos à criança/adolescente, é necessário que a família e os profissionais da saúde estejam preparadas para atuar frente à temática. O enfermeiro deve encorajar a comunicação dos cuidadores e o reconhecimento dos esforços da criança/adolescente para aprender a gerenciar as informações sobre a sua condição.

Em relação a medicação antirretroviral sob o olhar da criança/adolescente, principalmente durante a primeira fase da infância, pode influenciar de forma negativa na adesão ao tratamento. Encontram grande dificuldade em administrar os antirretrovirais, pois são medicações que geralmente causam reações gástricas como dor no estômago, enjoo e vômitos, sendo difícil a sua ingestão. Sua maior preocupação é que a medicação deverá ser utilizada por toda a vida. Os efeitos indesejáveis, como náuseas, dores de estômago e mal-estar também foram observados como barreiras para uma boa adesão a terapia ${ }^{13}$.

Um dos obstáculos que dificultam a adesão a terapia antirretroviral é referente ao horário da sua administração o que pode causar a interrupção do ciclo do sono, além da necessidade de acordar cedo ou dormir tarde para tomar os remédios. Além disso, constatou-se que as medicações podem impor algumas restrições, tornando dificultosa a adesão, principalmente na adolescência, na qual os adolescentes, muitas vezes, privam-se de atividades como viagens ou festas com os amigos ${ }^{13}$

Verificou-se que a má adesão medicamentosa ocorre devido ao baixo nível educacional, a falta ou ao pouco suporte familiar e social, ao consumo de bebida alcoólica e drogas ilícitas, além dos efeitos colaterais ao uso dos medicamentos. Assim, percebe-se que a equipe de enfermagem pode atuar com a comunidade e nas instituições de apoio de forma a orientar e trabalhar com as pessoas que necessitam de auxilio, seja em suas atividades diárias, na distribuição ou administração do TARV ${ }^{14}$.

$\mathrm{O}$ apoio e o auxílio fornecido às famílias que possuem crianças/adolescentes com HIV são essenciais para que esse grupo consiga se organizar e atender as necessidades da criança/adolescente com qualidade, principalmente em relação a rotina medicamentosa. A presença de um profissional que auxilie por meio das intervenções adequadas, que seja presente e forneça suporte é importante para que o familiar tenha condições de passar por esse processo.

Um estudo realizado, com crianças em um ambulatório de atendimento especializado em Infeç̧ões Sexualmente Transmissíveis (IST), constatou que os participantes revelaram hábitos alimentares restritivos, o que inibe a ingestão de alimentos que são essenciais para um bom desenvolvimento ${ }^{15}$. Além disso, a quantidade de medicações que são ingeridas também interfere nos hábitos alimentares da criança/adolescente, causando grande preocupação no familiar cuidador ${ }^{8}$.

Observou-se também que mesmo a alimentação sendo importante para auxiliar na qualidade de vida dos pacientes com HIV, os adolescentes soropositivos possuem um padrão alimentar parecido com os adolescentes sem a doença. Fator esse preocupante, visto que a maioria dos adolescentes soropositivos possuem maior chance de desenvolver doenças crônicas. Assim, é preciso que esses mantenham uma dieta nutritiva e adequada para sua faixaetária, além do acompanhamento periódico aos serviços de saúde e nas consultas com a equipe multidisciplinar ${ }^{16}$.

O enfermeiro é uma figura importante e durante as consultas com a família e a criança/adolescente deve estimular hábitos alimentares saudáveis, além de criar estratégias que estimulem a implementação de uma alimentação adequada, dentro das condições e da individualidade de cada família. A educação em saúde, as orientações, a promoção e prevenção são ações que fazem parte do cuidado de enfermagem e necessitam alcançar esses grupos de forma a auxiliá-los. 
Ao cuidar de uma criança/adolescente seu responsável necessita assumir seu cuidado, ainda mais quando a mesma é portadora de uma doença que exige cuidados específicos. A adesão à terapia medicamentosa não depende somente da criança/adolescente, mas também da responsabilidade e dos cuidados despendidos pelo familiar cuidador $^{17}$. Logo, o mesmo necessita adaptar suas rotinas e utilizar as estratégias aprendidas como forma de auxílio para que as dificuldades encontradas sejam sanadas.

A necessidade de adquirir responsabilidades e desempenhar um cuidado adequado que garanta o sucesso do tratamento é essencial para que a criança/adolescente tenha qualidade de vida. Nesse sentido, o familiar cuidador precisa compreender a importância do seu papel dentro da vida da criança/adolescente com HIV e assumir responsabilidade frente às demandas que surgirão. Para isso, a presença de um enfermeiro que escute e entenda a realidade dessa família é imprescindível para auxiliar na sua compreensão acerca da sua importância no cuidado com a criança/adolescente.

Verificou-se que situações referentes ao preconceito e a aceitação podem ocorrer dentro do ambiente escolar da criança/adolescente, levando muitas vezes a omissão do diagnóstico por medo que a mesma sofra humilhações e preconceito pelos próprios professores. Outro problema enfrentado pelo familiar cuidador é devido ao receio que as pessoas têm de contrair a doença ao entrar em contato com alguém soropositivo, causando dificuldades em encontrar ajuda para cuidar da criança/adolescente ${ }^{18}$

Além disso, a doença pode refletir negativamente na vida da criança/adolescente, devido aos estigmas e ao preconceito. Observou-se que devido ao desconhecimento das pessoas sobre a doença o familiar cuidador mencionou que a criança/adolescente sofria rejeição pelos colegas não participando das brincadeiras na escola ${ }^{19}$. Essa falta de conhecimento ainda existente muitas vezes faz com que o portador se silencie sobre a doença, enfrentando discriminação que pode ocorrer até mesmo por parte da própria família, vizinhos e até mesmo de profissionais da saúde. Este tipo de situação pode afetar a autoestima do indivíduo interferindo no seu autocuidado ${ }^{12}$. Portanto, a inclusão do indivíduo com HIV deve implicar na produção de um cuidado que desconstrua formas de preconceito e ofereça uma perspectiva de vida e de futuro com mais qualidade ${ }^{12}$.

Além disso, os profissionais da saúde e órgãos governamentais necessitam investir em campanhas e em práticas que estimulem o conhecimento da população sobre o HIV para que os indivíduos soropositivos vivam como qualquer outro cidadão. Além disso, o enfermeiro deve atuar na promoção do pensamento crítico desses pacientes diante das potencialidades e fragilidades, para que eles se sintam fortalecidos e confiantes para enfrentar as dificuldades e a incompreensão ainda existente da sociedade.

\section{CONCLUSÃO}

Verificou-se que as dificuldades apresentadas são muitas e interferem na vida e na rotina tanto da família quanto da criança/adolescente com HIV. Referiram dificuldades em manter o sigilo do diagnóstico para a criança/adolescente; na adesão desta à medicação e minimização dos seus efeitos colaterais, em seguir os horários de administração; na assiduidade às consultas; em sofrer preconceito e na aceitação da sociedade diante da doença, referiram, também, preocupação com o futuro da criança/adolescente.

Os dados possibilitaram concluir a importância da enfermagem como parte atuante e integrante da equipe multidisciplinar, visto que é ela quem entra primeiro em contato com esses pacientes e suas famílias tanto na Atenção Primária de Saúde como nos serviços especializados. O enfermeiro se faz presente realizando orientações, educação em saúde e na humanização da assistência, visando à promoção e a qualidade de vida da criança/ adolescente com HIV e sua família. Assim, frente a essa atuação será capaz de criar estratégias, de acordo com a individualidade e vivência de cada família, a fim de que elas consigam prestar um cuidado de qualidade a criança/adolescente, minimizando as dificuldades vivenciadas no seu dia a dia.

\section{REFERÊNCIAS}

1. Motta MGC, Ribeiro AC, Poletto, PMB, Issi HB, Ribeiro NRR, Padoin SMM. Family care in the child and the adolescent world living with HIV/Aids. Cienc. Enferm. 2014 [cited 2019 Apr 19]; 20 (3): 69-79. DOI: http://dx.doi.org/10.4067/S071795532014000300007

2. Potrich T, Paula CC, Padoin SMM, Gomes AMT. Relatives' day-to-day experience of caring for HIV-positive children in antiretroviral treatment. Rev Enferm UERJ. 2016 [cited 2019 Apr 19]; 24 (4): e17446. DOI: http://dx.doi.org/10.12957/reuerj.2016.17446

3. Alvarenga WA, Galvão MTG, Nascimento LC, Beretta MI, Dupas G. Weakened social network: the experience of caregivers of the HIV-exposed infant. Texto Contexto - Enferm. 2015 [cited 2019 Apr 19]; 24 (3): 775-83. DOI:

http://dx.doi.org/10.1590/0104-07072015011160014 
4. Lorenz R, Grant E, Muyindike W, Maling S, Card C, Henry C, et al. Caregivers' Attitudes towards HIV Testing and Disclosure of HIV Status to At-Risk Children in Rural Uganda. PLoS ONE. 2016 [cited 2019 Apr 19]; 11(2): e0148950. DOI: https://doi.org/10.1371/journal.pone.0154169

5. Koerich C, Santos FC, Meirelles BHS, Erdmann AL. Management of nursing care of the adolescent living with HIV/AIDS. Esc. Anna Nery Rev. Enferm. 2015 [cited 2019 Apr 19]; 19 (1):115-23. Available from: http://www.scielo.br/pdf/ean/v19n1/1414-8145ean-19-01-0115.pdf

6. Bardin L. Análise de Conteúdo. 70 ạed. Lisboa; 2011.

7. Kajubi $P$, Whyte SR, Kyaddondo D, Katahoire AR. Tensions in Communication between Children on Antiretroviral Therapy and Their Caregivers: A Qualitative Study in Jinja District, Uganda. PLos ONE. 2016 [cited 2019 Apr 19]; 11 (1):1-21. DOI: https://doi.org/10.1371/journal.pone.0147119

8. Pacheco BP, Gomes GC, Xavier DM, Nobre CMG, Aquino DR. Difficulties and facilities of the family to care for children with HIV/Aids. Esc Anna Nery. 2016 [cited 2019 Apr 19]; 20 (2): 378-83. Available from: http://www.scielo.br/pdf/ean/v20n2/14148145-ean-20-02-0378.pdf.

9. Zanon BP, Paula CC, Padoin SMM. Revealing an HIV diagnosis for children and adolescents: subsidy for the practice of care. Rev Gaúcha Enferm. 2016 [cited 2019 Apr 19]; 37 (n.esp):e2016-0040. DOI: http://dx.doi.org/10.1590/1983-1447.2016.esp.20160040

10. Borges JMC, Pinto JA, Ricas J. Children and adolescents living with hiv/aids: which disease is that? Reverso. 2015 [cited 2019 Apr 19]; 37 (70): 67-74. Available from: http://pepsic.bvsalud.org/scielo.php?script=sci_arttext\&pid=S0102-73952015000200009

11. Lima MCD. Social service and accession to the treatment of children and teens seropositive: experience report. Divers@. 2015 [cited 2019 Apr 19]; 8 (1): 43-50. DOI: http://dx.doi.org/10.5380/diver.v8i1.43876

12. Sehnem GD, Brondani JP, Kantorski KJC, Silva SC, Ressel LB, Pedro ENB. Health in adolescence with HIV / AIDS: paths for a post2015 schedule. Rev. Gaúcha Enferm. 2015 [cited 2019 Apr 19]; 36 (n. esp): 39-46. DOI: http://dx.doi.org/10.1590/19831447.2015.esp.57385

13. Galano E, Turato ER, Delmas P, Côté J, Gouvea AFTB, Succi RCM, et al. Experiences of adolescents seropositive for HIV/AIDS: a qualitative study. Rev. Paul Pediatr. 2016 [cited 2019 Apr 19]; 34 (2): 171-7. DOI: http://dx.doi.org/10.1016/j.rppede.2015.08.019

14. Santos El, Silva AL, Santana PPC, Barros CHA, Assis CT, Nogueira PM, et al. Evidências científicas brasileiras sobre adesão à terapia antirretroviral por pessoas que vivem com HIV. Rev. Rede Cuid. Saúde. 2016 [cited 2019 Apr 19]; 10 (1):1-15. Available from: http://publicacoes.unigranrio.edu.br/index.php/rcs/article/view/2554

15. Poletto PMB, Motta MGC. Education in health in the waiting room: care and actions to the child who lives with HIV/aids. Esc. Anna Nery. 2015 [cited 2019 Apr 19]; 19 (4): 641-7. Available from: http://www.scielo.br/scielo.php?pid=S1414$81452015000400641 \&$ script=sci_abstract\&tlng=pt

16. Tanaka LF, Silva AM, Konstantyner TCRO, Mendes EC, Marques HHS. Poor diet quality among Brazilian adolescents with HIV/AIDS. J Pediatr. 2015 [cited 2019 Apr 19]; 91(2):152-9. DOI: http://dx.doi.org/10.1016/j.jped.2014.06.007

17. Favero NB, Sehnem GD, Silveira A, Stenert F. Anti-retroviral therapy in the daily life of adolescents with HIV/Aids. Cienc. Enferm. 2016 [cited 2019 Apr 19]; 22 (1): 23-33. DOI: http://dx.doi.org/10.4067/S0717-95532016000100003

18. Santos El, Silva AL, Santana PPC, Barros CHA, Assis CT, Nogueira PM, et al. Brazilian scientific evidences about adherence to antiretroviral therapy by patients with HIV/Aids. Rev. Rede Cuid. Saúde. 2016 [cited 2019 Apr 19]; 10 (1):1-15. Available from: http://publicacoes.unigranrio.edu.br/index.php/rcs/article/view/2554

19. Lara MM, Gomes GC, Nobre CMG, Jung BC, Costa AR, Rodrigues EF. Perception of the family caregiver regarding problems faced by children in relation to their diagnosis of HIV/aids. Cogitare Enferm. 2017 [cited 2019 Apr 19]; 22, n. 4, p.e50882, 2017. DOI: http://dx.doi.org/10.5380/ce.v22i4.50882 